\title{
Serum fibrosis index-based risk score predicts hepato- cellular carcinoma in untreated patients with chronic hepatitis B
}

Lilian Yan Liang ${ }^{1,2,3, *}$, Hye Won Lee ${ }^{4, *}$, Vincent Wai-Sun Wong ${ }^{1,2,3}$, Terry Cheuk-Fung Yip ${ }^{1,2,3}$, Yee-Kit Tse ${ }^{1,2,3}$, Vicki Wing-Ki Hui ${ }^{1,2,3}$, Grace Chung-Yan Lui ${ }^{2,3}$, Henry Lik-Yuen Chan ${ }^{1,2,3}$, and Grace Lai-Hung Wong ${ }^{1,2,3}$

${ }^{1}$ Institute of Digestive Disease, ${ }^{2}$ Department of Medicine and Therapeutics, ${ }^{3}$ Medical Data Analytic Centre (MDAC), The Chinese University of Hong Kong, Hong Kong SAR; “Department of Internal Medicine, Yonsei University College of Medicine, Seoul, Korea

\section{Graphical Abstract}

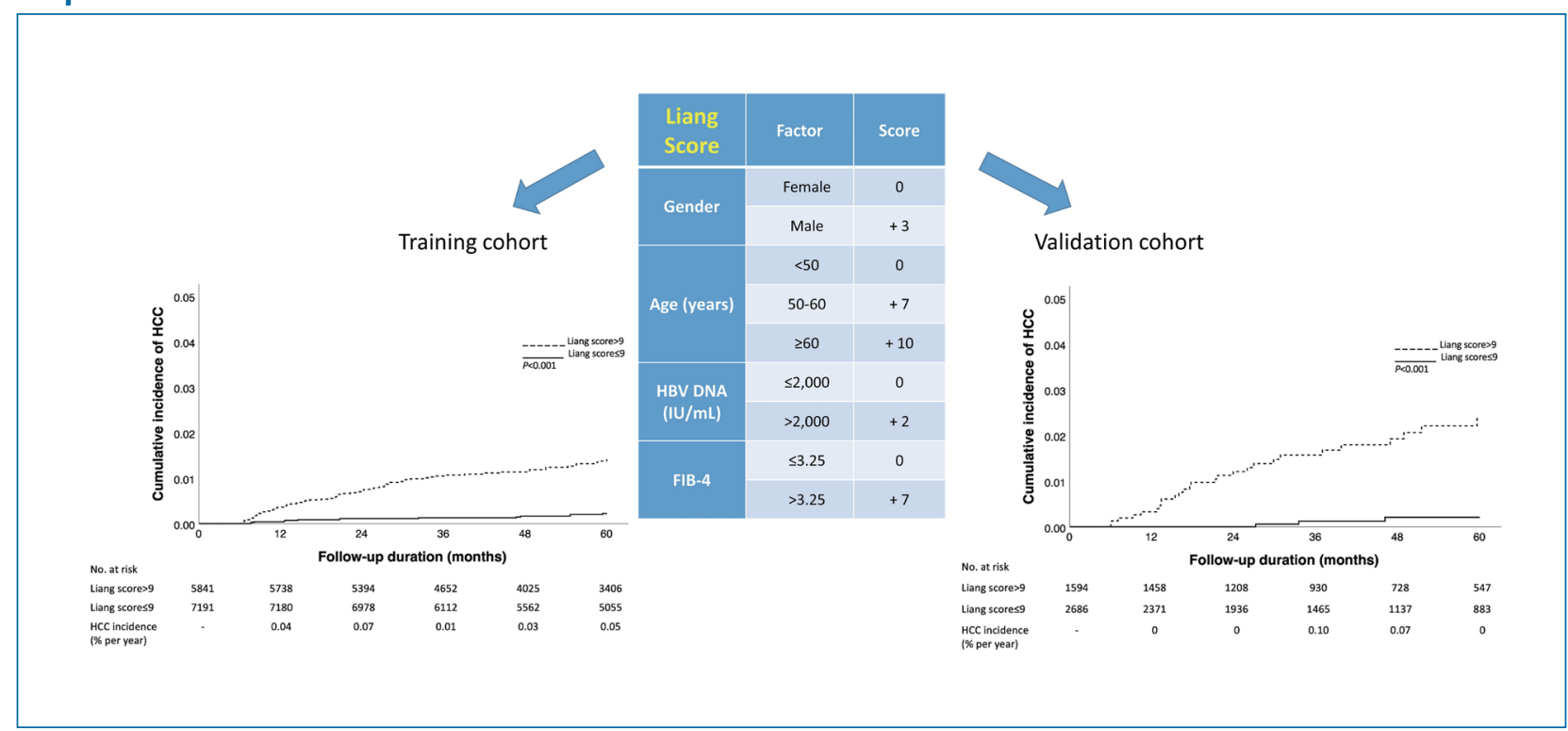

\section{Abbreviations:}

AFP, alpha-fetoprotein; ALT, alanine aminotransferase; APRI, aspartate aminotransferase to platelet ratio index; $\mathrm{AUROC}$, area under the time-dependent receiver operating characteristic curve; CDARS, the Clinical Data Analysis and Reporting System; $\mathrm{CHB}$, chronic hepatitis $\mathrm{B} ; \mathrm{Cl}$, confidence interval; $\mathrm{CU}-\mathrm{HCC}$, the Chinese University of Hong Kong-HCC; DM, diabetes mellitus; FIB-4, fibrosis-4; GAG-HCC, the guide with age, gender, HBV DNA, core promoter mutations and cirrhosis-HCC; GGT, gamma-glutaryl transpeptidase; HBeAg, hepatitis e antigen; HBV, hepatitis B virus; $\mathrm{HCC}$, hepatocellular carcinoma; ICD-9-CM, the International Classification of Disease, Ninth Revision, Clinical Modification; IQR, interquartile range; NPV, negative predictive value; REACH-B, the risk estimation for HCC in CHB; WHO, World Health Organization

\section{Corresponding author : Grace Lai-Hung Wong}

Department of Medicine and Therapeutics, The Chinese University of Hong Kong, 9/F., Lui Che Woo Clinical Sciences Building, Shatin, N.T., Hong Kong

Tel: +852-2632-3538, Fax: +852-2637-3852

E-mail:wonglaihung@cuhk.edu.hk

https://orcid.org/0000-0002-2863-9389

*Liang LY and Lee HW have equal contribution to this manuscript. 
Background/Aims: Serum fibrosis scores comprised of common laboratory tests have high utility to assess severity of liver fibrosis. We aimed to derive and validate a hepatocellular carcinoma (HCC) risk score based on serum fibrosis scores to predict HCC in treatment-naïve chronic hepatitis B (CHB) patients.

Methods: Fifteen thousand one hundred eighty-seven treatment-naive adult CHB patients were identified to form the training cohort in this retrospective study. Individual fibrosis score was included to construct a new HCC prediction score. The score was externally validated in an independent treatment-naïve Korean $\mathrm{CHB}$ cohort.

Results: $180 / 15,187$ patients (1.2\%) in training cohort and 47/4,286 patients (1.1\%) in validation cohort developed HCC during a mean follow-up of 52 and 50 months, respectively. The newly developed HCC risk score, Liang score, is composed of gender, age, hepatitis B virus DNA, fibrosis-4 (FIB-4) index, and ranges from 0 to 22 . Area under the timedependent receiver operating characteristic curve of Liang score was 0.79 ( $95 \%$ confidence interval, $0.70-0.89$ ). A cutoff value of nine provided an extremely high negative predictive value of $99.9 \%$ and high sensitivity of $90.0 \%$ at 5 years in the validation cohort. Patients with Liang score $\leq 9$ had HCC incidence $<0.2 \%$ per year in both training and validation cohorts, in whom HCC surveillance might be exempted.

Conclusion: A novel HCC risk score, Liang score, based on FIB-4 index, is applicable and accurate to identify treatmentnaïve CHB patients with very low risk of HCC to be exempted from HCC surveillance. (Clin Mol Hepatol 2021;27:499509)

Keywords: Hepatitis B, Chronic; Carcinoma, Hepatocellular; Fibrosis; Surveillance

\section{Study Highlights}

Serum fibrosis scores comprised of common laboratory tests have high utility to assess severity of liver fibrosis which is correlated with the risk of HCC. The newly developed HCC risk score, Liang score, is composed of gender, age, HBV DNA, FIB-4 index and had high sensitivity and negative predictive value to predict HCC of treatment-naive CHB patients. Liang score is accurate to identify treatment-naive CHB patients with very low risk of HCC to be exempted from HCC surveillance.

\section{INTRODUCTION}

Chronic hepatitis B virus (HBV) infects approximately 292 million persons around the world and attributes to about half of the hepatocellular carcinoma (HCC) cases every year. ${ }^{1} \mathrm{HCC}$ is a cancer with high mortality rate, with most cases detected at advanced stages. ${ }^{2}$ HCC surveillance remains indispensable to detect tumor at early stages in order to improve the prognosis of patients. ${ }^{3}$ Chronic hepatitis B (CHB) patients with high serum HBV DNA levels, elevated alanine aminotransferase (ALT) levels, advanced fibrosis and necroinflammation are at risk of developing HCC, hence are indicated for antiviral therapy. ${ }^{4 \cdot 6} \mathrm{CHB}$ patients received antiviral treatment have significantly lower risk of HCC and liverrelated mortality. ${ }^{78}$ On the other hand, patients who do not fulfil treatment criteria may also develop HCC. ${ }^{9,10}$ Thus, some untreated patients may have a significant risk of HCC development and thus benefit from antiviral treatment.

Advanced fibrosis and cirrhosis are the key risk factors of HCC. ${ }^{11,12}$ Liver biopsy examination was commonly used to assess liver fibrosis, yet its invasiveness and potential complications limit its generalizability. ${ }^{13}$ Liver stiffness measurement by transient elastography is a non-invasive method to assess liver fibrosis, yet the measurement is limited by the availability of the machine. Instead, various serum test-based formulae have been developed to predict liver fibrosis with a reasonable accuracy. While some of these formulae (for example Enhanced Liver Fibrosis) require the measurement of uncommon and expensive laboratory parameters including aminoterminal propeptide of type III procollagen, hyaluronic acid and tissue inhibitor of matrix metalloproteinase type1. ${ }^{14} \mathrm{~A}$ few formulae comprised of common laboratory tests have been widely used. The most commonly used formulae include aspartate aminotransferase to platelet ratio index (APRI), fibrosis-4 (FIB-4) index, and Forns index. ${ }^{15}$ These serum fibrosis scores that reflected the severity of liver fibrosis could be useful to define the HCC risk of CHB patients, in particulars in those remained untreated. ${ }^{16,17}$ Existing HCC risk scores for untreated CHB patients including the Chinese University of Hong Kong-HCC (CU-HCC) score, the guide with age, gender, HBV DNA, core promoter mu- 
tations and cirrhosis-HCC (GAG-HCC) score (without core promoter mutation), and the risk estimation for HCC in CHB (REACH-B) score rely heavily on clinical diagnosis of cirrhosis. The clinical diagnosis can however be subjective and vary among centers as liver biopsy is not performed in most of the patients. Any misdiagnosis of liver cirrhosis will affect much on the classification of patients to different risk groups.

Based on the recommendations of American Association for the Study of Liver Diseases, an annual HCC risk of $<0.2 \%$ is probably not cost-effective for HCC surveillance. ${ }^{4}$ In view of the huge number of CHB patients, a prediction score with high sensitivity to identify patients at very low risk of HCC would be pivotal to appropriate allocation of healthcare resources for HCC surveillance. We may exempt these very-low-risk patients from HCC surveillance and offer regular monitoring at primary care setting instead of secondary or tertiary centers. ${ }^{6}$ Appropriate resource allocation facilitates the elimination of HBV infection by 2030, a goal set by the World Health Organization (WHO). ${ }^{18}$ In the current study, we aimed to derive and validate a $\mathrm{HCC}$ risk score developed based on serum test formulae to exclude treatment-naïve CHB patients with low HCC risk.

\section{PATIENTS AND METHODS}

\section{Study design and data source}

The training cohort was a territory-wide retrospective cohort identified using data from the Clinical Data Analysis and Reporting System (CDARS) under the management of Hospital Authority, Hong Kong. ${ }^{19}$ Different territory-wide studies have been conducted using CDARS..$^{20-23}$ The International Classification of Diseases, Ninth Revision, Clinical Modification (ICD-9-CM) coding system was used in CDARS. The use of ICD-9-CM codes in CDARS to identify hepatic events has been found $99 \%$ accurate when referenced to clinical, laboratory, imaging and endoscopy results from the electronic medical records. ${ }^{24}$ The derived HCC risk score was externally validated in an independent cohort of CHB patients from Severance Hospital, Seoul, Korea.

\section{Subjects}

Consecutive adult CHB patients with positive hepatitis B surface antigen for 6 months and/or defined by the ICD-9-CM diagnosis codes between 2000 and 2017 in Hong Kong were identified.
Those patients with serum HBV DNA and at least one of the serum fibrosis scores (APRI, FIB-4, and Forns index) measured between January 2000 and June 2018 were included. Follow-up duration were censored at the start date of first antiviral treatment for patients ever received antiviral treatment. HCC surveillance was done every 6 to 12 months by ultrasound and alpha-fetoprotein (AFP) testing under the Hospital Authority. APRI, FIB-4, and Forns index were calculated as described (Supplementary Table 1). ${ }^{15,25,26}$ We excluded the following patients: those co-infected with hepatitis C virus and/or hepatitis D virus based on ICD-9-CM diagnosis codes, viral and serological markers and/or use of antiviral therapy for hepatitis $C$; co-infected with human immunodeficiency virus based on ICD-9-CM diagnosis codes and/or use of antiviral therapy for human immunodeficiency virus; had other coexisting autoimmune and metabolic liver diseases based on ICD9-CM diagnosis codes; had cancer including HCC and/or liver transplantation before or within the first 6 months after baseline date; follow-up duration less than 6 months; received rituximab and any chemotherapeutic agents (Supplementary Tables 1, 2). We also excluded patients with ICD-9-CM diagnosis codes of acute hepatitis $B$ and/or positive immunoglobulin $\mathrm{M}$ to hepatitis $\mathrm{B}$ core antigen unless it was more than 6 months apart from a positive hepatitis B surface antigen result, patients with Child-Pugh class $B$ or $C$ or had any hepatic events before baseline. For validation cohort, CHB patients identified in 2000-2017 with available HBV DNA results in Severance Hospital, Seoul, Korea were included. We excluded patients age younger than 18 years; develop HCC before or within first 6 months after baseline; follow-up less than 6 months; without available serum fibrosis scores at baseline; with Child Pugh B or C and/or had any hepatic events before baseline.

Patients were followed until diagnosis of HCC, death, liver transplantation, starting antiviral treatment, last follow-up date (December 31, 2018), or up to 5 years of follow-up, whichever came first. The study protocol was approved by the Joint Chinese University of Hong Kong - New Territories East Cluster Clinical Research Ethics Committee.

\section{Data collection}

Data of training cohort were retrieved from CDARS in January 2019. Baseline date was set on the first serum HBV DNA assay. Demographic data including date of birth and gender were captured. At baseline, liver and renal biochemistries, hematological and virologic parameters were collected. Thereafter, serial liver 


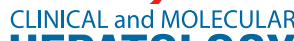

\section{HEPATOLOGY}

and renal biochemistries as well as HBV viral markers were collected until the last follow-up date. We also retrieved data on other relevant diagnoses, procedures, concomitant drugs, laboratory parameters. Drug data of any antiviral treatment for CHB including any approved nucleos(t)ide analogues, namely lamivudine, adefovir dipivoxil, entecavir, telbivudine, tenofovir disoproxil fumarate and tenofovir alafenamide, as well as (pegylated)-interferon were also retrieved during the study period; patients' followup was censored at the time of first antiviral treatment.

\section{Definitions}

The primary endpoint was HCC. HCC was identified based on diagnosis codes (155.0: HCC and 155.2: carcinoma of liver) or procedure codes for HCC treatment according to ICD-9-CM codes from CDARS. Liver cirrhosis was identified by ICD-9-CM diagnosis codes for cirrhosis and its related complications (Supplementary Table 2). Diabetes mellitus (DM) was defined by exposure to any anti-diabetic agents, and/or hemoglobin A1c $\geq 6.5 \%$ and/or fasting plasma glucose $\geq 7.0 \mathrm{mmol} / \mathrm{L}$ in one measurement, and/or the ICD-9-CM diagnosis codes for DM.

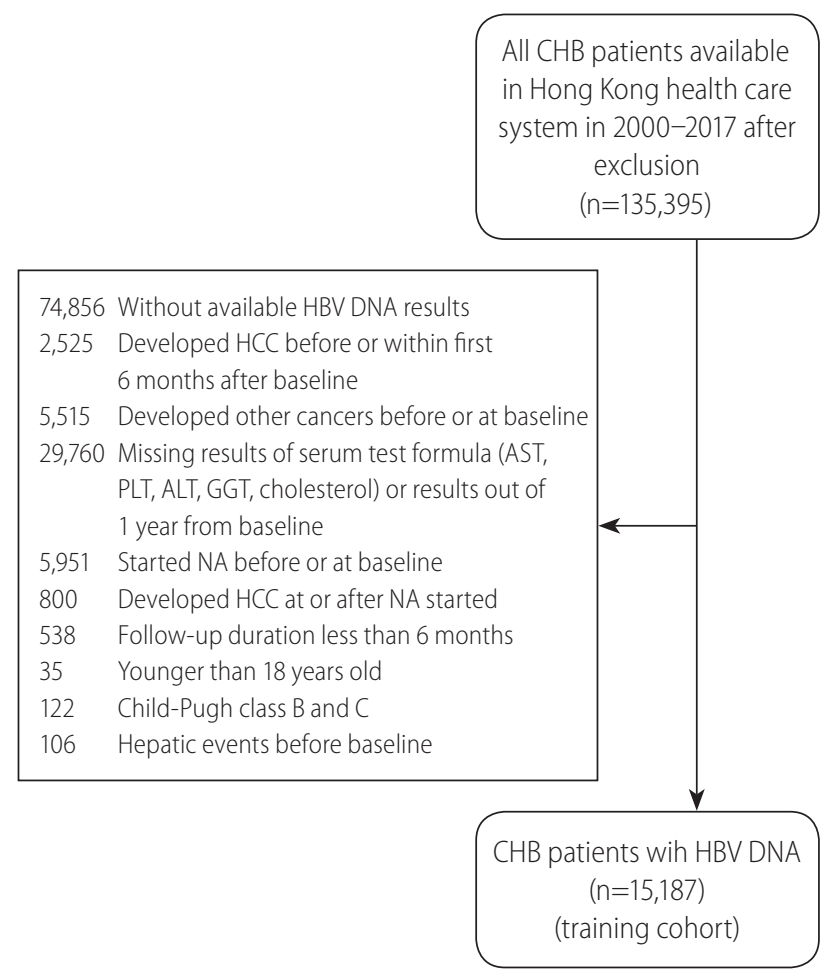

\section{Statistical analysis}

Data were analyzed using SPSS version 25.0 (IBM Corp., Armonk, NY, USA), and R software (4.0.0; R Foundation for Statistical Computing, Vienna, Austria). Continuous variables were expressed in mean \pm standard deviation or median (interquartile range $[\mathrm{IQR}])$, as appropriate, while categorical variables were presented as frequency (percentage). Time to HCC diagnosis was defined as the time from the baseline date to the date of first HCC diagnosis code, or the date of last follow-up if patients were still alive without HCC at the time of analysis. Kaplan-Meier analysis with log-rank test was used to estimate and compare the cumulative risks of development of HCC in different groups of patients. Cox proportional hazards model was performed to determine the relationship of clinical characteristics of HBV DNA level, gender, age, serum bilirubin level, FIB-4 index and serum hepatitis e antigen ( $\mathrm{HBeAg}$ ) status with the development of HCC. Missing values were excluded from the analysis. A simple HCC risk score was derived by significant variables obtained from backward stepwise multivariable analysis with $P<0.05$. The score was the weighted sum of those variables of which the weights were defined as the

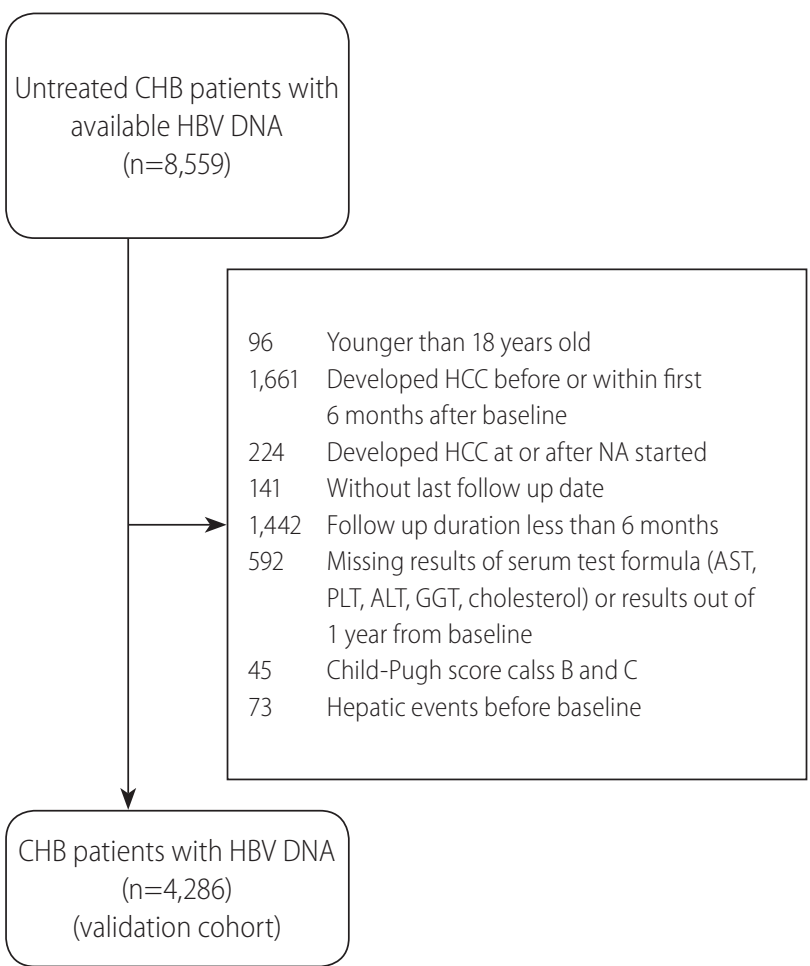

Figure 1. The selection of patients in the training cohort and validation cohort. CHB, chronic hepatitis B; HBV, hepatitis B virus; HCC, hepatocellular carcinoma; AST, aspartate amionotransferase; PLT, platelet; ALT, alanine aminotransferase; GGT, gamma-glutaryl transpeptidase; NA, nucleos(t)ide analogues. 
Lilian Yan Liang, et al. Fibrosis scores and hepatocellular carcinoma risk

quotient (rounded to nearest integer) of corresponding estimated coefficient from a Cox regression analysis divided by the smallest chi-square coefficient."

The performance of serum fibrosis scores and the HCC risk score was assessed by area under the time-dependent receiver operating characteristic curve (AUROC). ${ }^{27}$ The $95 \%$ confidence interval (CI) for the comparison of two ROC curves was computed by bootstrap sampling of 1,000 samples. Diagnostic accuracy of the suggested cutoff values was assessed by sensitivity, specificity, positive predictive value, and negative predictive value (NPV) with $95 \% \mathrm{Cl}$. Youden's index was used to select the best cutoff value. High sensitivity and NPV were used to further select the cutoff values with comparable Youden's index to exclude patients with low HCC risk. All statistical tests were two-sided. $P<0.05$ was taken as statistically significant.

\section{RESULTS}

\section{Patients characteristics}

We identified in total 135,395 CHB patients in the public healthcare system in 2000-2017. ${ }^{28}$ There were 60,539 CHB patients with available HBV DNA results at baseline. After excluding patients fulfilling the exclusion criteria, the final number of treatment-naïve CHB patients included in the training cohort was 15,187. We identified 8,559 untreated CHB patients with available HBV DNA measurement in the validation cohort, 4,286 patients were finally analyzed after exclusion (Fig. 1).

During a mean follow-up of 52 months, 180 patients developed HCC in the training cohort. We compared the characteristics of patients developed HCC and without HCC. Patients who devel-

Table 1. Clinical characteristics of patients in the training and validation cohorts

\begin{tabular}{|c|c|c|c|c|c|c|}
\hline & \multicolumn{3}{|c|}{ Training cohort } & \multicolumn{3}{|c|}{ Validation cohort } \\
\hline & $\begin{array}{c}\text { HCC } \\
(n=180)\end{array}$ & $\begin{array}{c}\text { Non-HCC } \\
(n=15,007)\end{array}$ & $P$-value & $\begin{array}{c}\text { HCC } \\
(n=47)\end{array}$ & $\begin{array}{l}\text { Non-HCC } \\
(n=4,239)\end{array}$ & $P$-value \\
\hline Male & $133(73.9)$ & $8,220(54.8)$ & $<0.001$ & $39(83.0)$ & $2,249(53.1)$ & $<0.001$ \\
\hline Age (years) & $61.4 \pm 11.4$ & $51.8 \pm 13.6$ & $<0.001$ & $58.0 \pm 11.9$ & $48.4 \pm 12.6$ & $<0.001$ \\
\hline Age $<50$ years & $24(13.3)$ & $6,295(41.9)$ & $<0.001$ & $10(21.3)$ & 2,228 (51.6) & $<0.001$ \\
\hline Albumin (g/L) & $38.1 \pm 5.6$ & $41.6 \pm 4.9$ & $<0.001$ & $39.5 \pm 5.3$ & $42.8 \pm 4.7$ & $<0.001$ \\
\hline Bilirubin ( $\mu \mathrm{mol} / \mathrm{L})$ & $14.1(9.2-19.1)$ & $11.0(8.0-15.8)$ & $<0.001$ & $13.7(10.3-18.8)$ & $12.0(10.3-17.1)$ & 0.964 \\
\hline ALT (IU/L) & $46.0(31.0-72.3)$ & $37.0(22.0-73.0)$ & 0.001 & $26.0(17.0-39.0)$ & $24.0(16.0-42.0)$ & 0.678 \\
\hline AST (U/L) & $33.1(24.0-52.2)$ & $30.0(23.0-49.0)$ & 0.081 & $30.0(24.0-53.0)$ & $24.0(19.0-36.0)$ & 0.001 \\
\hline Platelet $\left(\times 10^{9} / \mathrm{L}\right)$ & $161.3 \pm 68.2$ & $208.5 \pm 68.2$ & $<0.001$ & $155.1 \pm 63.2$ & $201.2 \pm 68.3$ & $<0.001$ \\
\hline GGT (U/L) & $70.2(37.0-164.5)$ & $29.0(19.0-58.0)$ & $<0.001$ & $56.5(24.0-100.5)$ & $25.0(16.0-46.0)$ & 0.001 \\
\hline Cholesterol (mmol/L) & $4.6 \pm 1.2$ & $4.8 \pm 1.1$ & 0.002 & $4.2 \pm 1.0$ & $4.7 \pm 1.0$ & 0.002 \\
\hline Positive HBeAg & $28(18.8)$ & $2,403(17.7)$ & 0.738 & $5(13.2)$ & $786(23.3)$ & 0.140 \\
\hline HBV DNA $\left(\log _{10} \mid \mathrm{I} / \mathrm{mL}\right)$ & $5.0 \pm 1.8$ & $4.4 \pm 2.1$ & $<0.001$ & $3.1 \pm 1.7$ & $3.5 \pm 2.1$ & 0.015 \\
\hline HBV DNA > 2,000 IU/mL & $138(76.7)$ & $8,711(58.0)$ & $<0.001$ & $11(23.4)$ & $1,374(32.4)$ & 0.189 \\
\hline Cirrhosis & $56(31.1)$ & 765 (5.1) & $<0.001$ & 37 (78.7) & $916(21.6)$ & $<0.001$ \\
\hline APRI & $0.7(0.5-1.2)$ & $0.4(0.3-0.7)$ & $<0.001$ & $0.5(0.4-0.9)$ & $0.3(0.2-0.6)$ & $<0.001$ \\
\hline FIB-4 index & $2.5(1.6-4.4)$ & $1.3(0.9-2.1)$ & $<0.001$ & $2.4(1.6-4.1)$ & $1.3(0.8-2.0)$ & $<0.001$ \\
\hline FIB-4 $>3.25$ & $54(38.6)$ & $1,611(12.5)$ & $<0.001$ & $18(38.3)$ & $471(11.1)$ & $<0.001$ \\
\hline Forns index & $7.4 \pm 2.0$ & $5.6 \pm 2.0$ & $<0.001$ & $6.8 \pm 2.1$ & $4.8 \pm 2.1$ & $<0.001$ \\
\hline $\begin{array}{l}\text { Advanced fibrosis (\%), APRI } \\
>2 / \text { FIB- } 4>3.25 / \text { Forns } \\
\text { index }>8.4\end{array}$ & $70(38.9)$ & 2,087 (13.9) & $<0.001$ & $20(42.6)$ & $537(12.7)$ & $<0.001$ \\
\hline Follow-up duration (months) & $48.9 \pm 34.8$ & $68.3 \pm 28.7$ & $<0.001$ & $46.0 \pm 32.0$ & $49.5 \pm 33.6$ & 0.477 \\
\hline
\end{tabular}

Values are presented as mean \pm standard deviation, median (interquartile range), or number (\%).

HCC, hepatocellular carcinoma; ALT, alanine aminotransferase; AST, aspartate amionotransferase; GGT, gamma-glutaryl transpeptidase; HBeAg, hepatitis B e antigen; HBV, hepatitis B virus; APRI, aspartate aminotransferase to platelet ratio index; FIB-4, fibrosis-4. 
oped HCC were older (61.4 \pm 11.4 vs. $51.8 \pm 13.6$ years), and more likely to be male $(73.9 \%$ vs. $54.8 \%)$ than patients without HCC. More patients of non-HCC group were younger than 50 years (41.9\%). For liver biochemistries, patients who developed HCC had lower albumin level $(38.1 \pm 5.6$ vs. $41.6 \pm 4.9 \mathrm{~g} / \mathrm{L})$, platelet count $\left(161.3 \pm 68.2\right.$ vs. $\left.208.5 \pm 68.2 \times 10^{9} / \mathrm{L}\right)$, cholesterol level (4.6 \pm 1.2 vs. $4.8 \pm 1.1 \mathrm{mmol} / \mathrm{L})$ and higher total bilirubin (14.1 [IQR,

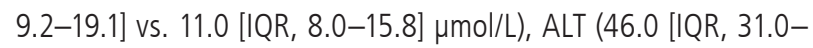
72.3] vs. 37.0 [IQR, 22.0-73.0] IU/L), gamma-glutaryl transpeptidase (GGT; 70.2 [IQR, 37.0-164.5] vs. 29.0 [IQR, 19.0-58.0] U/L) than patients without HCC. Patients with HCC had cirrhosis ( $31.1 \%$ vs. $5.1 \%$ ) and higher HBV DNA level ( $5.0 \pm 1.8$ vs. $4.4 \pm 2.1$ $\left.\log _{10} \mathrm{IU} / \mathrm{mL}\right)$. Their serum fibrosis scores of APRI (0.7 [IQR, $0.5-$ 1.2] vs. 0.4 [IQR, 0.3-0.7]), FIB-4 index (2.5 [IQR, 1.6-4.4] vs. 1.3 [IQR, 0.9-2.1]), and Forns index (7.4 \pm 2.0 vs. $5.6 \pm 2.0)$ were all higher than those of non-HCC patients (Table 1). The AFP levels in patients developed HCC was significantly higher than patients did not develop HCC (6.1 [IQR, 3.3-11.6] vs. 3.1 [IQR, 2.2-4.9] ng/mL, $P<0.001)$. Patients developed HCC had more proportion of DM than patients did not develop HCC (37.2\% vs. 18.6\%, $P<0.001)$.

In the validation cohort, 47 patients developed HCC during a mean follow-up of 50 months. Compared with the training cohort, patients in the validation cohort were younger $(48.5 \pm 12.6$ vs. $52.0 \pm 13.6)$ and had more patients with cirrhosis (22.2\% vs. $5.4 \%)$. Patients had similar albumin level, platelet count, APRI and FIB-4 index in both of the training and validation cohorts (Table 1).

\section{Predictors of HCC}

We performed univariate and multivariable analysis for APRI, FIB-4 index separately. Forns index is not generally used in clinical assessment of Hong Kong and there were more than half of missing values in our cohort. So, we did not select Forns index to develop the new score. APRI $>2$ was not associated with HCC development in both univariate and multivariable analysis (Supplementary Table 3). FIB-4 $>3.25$, male gender, age older than 50 years old, high total bilirubin (bilirubin $>18 \mu \mathrm{mol} / \mathrm{L}$ ), low platelet (platelet $<150 \times 10^{9} / \mathrm{L}$ ), high HBV DNA level (HBV DNA $>2,000 \mathrm{IU} / \mathrm{mL}$ ) and DM were correlated with HCC development by univariate analysis. ${ }^{29}$ There were 2,155 patients (14.2\%) without FIB-4 index and these patients were excluded from the Cox proportional hazards model. Male gender, age older than 50, high HBV DNA level and high FIB-4 could predict HCC development significantly in multivariable analysis (Table 2).

\section{Derivation of HCC prediction score}

FIB-4 score combined with gender, age and HBV DNA level which were significant in the multivariable analysis comprised the new HCC prediction score named Liang score and ranged from 0 to 22 (Table 3).

We listed out several potential cutoff values and calculated the diagnostic accuracy of these values (Table 4). Among these val-

Table 2. Univariate and multivariable analysis of Cox proportional hazards models on risk factors associated with hepatocellular carcinoma in the training cohort (follow-up duration: 5 years)

\begin{tabular}{|c|c|c|c|c|c|c|c|c|}
\hline \multirow{2}{*}{ Factor } & \multirow{2}{*}{$\begin{array}{c}\text { No. of } \\
\text { patients }\end{array}$} & \multicolumn{3}{|c|}{ Univariate } & \multicolumn{4}{|c|}{ Multivariable } \\
\hline & & HR & $95 \% \mathrm{Cl}$ & $P$-value & $\chi^{2}$ score & Adjusted HR & $95 \% \mathrm{Cl}$ & $P$-value \\
\hline Male sex & $8,353(55.0)$ & 1.96 & $1.31-2.95$ & 0.001 & 6.71 & 1.96 & $1.18-3.26$ & 0.010 \\
\hline \multicolumn{9}{|l|}{ Age (years) } \\
\hline$<50$ & 6,319 (41.6) & 1 & & & & 1 & & \\
\hline $50-60$ & $4,833(31.8)$ & 5.64 & $2.83-11.23$ & $<0.001$ & 15.89 & 4.93 & $2.25-10.79$ & $<0.001$ \\
\hline$\geq 60$ & 4,035 (26.6) & 10.61 & $5.44-20.71$ & $<0.001$ & 21.32 & 6.51 & $2.94-14.43$ & $<0.001$ \\
\hline Bilirubin $>18 \mu \mathrm{mol} / \mathrm{L}$ & 2,537 (16.7) & 2.00 & $1.33-3.01$ & 0.001 & & & & \\
\hline Platelet $<150 \times 10^{9} / \mathrm{L}$ & $2,654(17.5)$ & 3.40 & $2.34-4.95$ & $<0.001$ & & & & \\
\hline HBV DNA >2,000 IU/mL & $8,849(58.3)$ & 2.07 & $1.36-3.15$ & 0.001 & 4.26 & 1.77 & $1.03-3.03$ & 0.039 \\
\hline FIB-4 $>3.25$ & $1,667(11.0)$ & 4.46 & $2.89-6.88$ & $<0.001$ & 15.16 & 2.70 & $1.64-4.45$ & $<0.001$ \\
\hline HBeAg (+) & $2,431(16.0)$ & 0.86 & $0.49-1.49$ & 0.586 & & & & \\
\hline DM & 2,865 (18.9) & 3.14 & $2.16-4.58$ & $<0.001$ & & & & \\
\hline
\end{tabular}

Values are presented as number (\%) unless otherwise indicated.

HR, hazard ratio; Cl, confidence interval; HBV, hepatitis B virus; FIB-4, fibrosis-4; HBeAg, hepatitis B e antigen; DM, diabetes mellitus. 
ues, cutoff value of 10 had the highest Youden's index of 0.406 , followed by the cutoff value of 9 with Youden's index of 0.391 . As the corresponding sensitivity and NPV of 9 were $83.7 \%(95 \% \mathrm{Cl}$, 73.9-90.5\%) and 99.8\% (95\% Cl, 99.7-99.9\%), which were higher than those of the cutoff value of 10 (sensitivity, 74.4\%; specificity, 99.7\%), we finally selected 9 as the cutoff value of the Liang score. By using the cutoff value of 9 , patients were divided into two groups: low-risk group (7,191 patients) with Liang score $\leq 9$ and high-risk group (5,841 patients) with Liang score $>9$. The corresponding number of patients developed HCC were 14 $(0.19 \%)$ and $72(1.12 \%)$ in the low- and high-risk groups at

Table 3. Components of the Liang score

\begin{tabular}{lc}
\hline Factor & Score \\
\hline Gender & 0 \\
Female & +3 \\
Male & \\
Age (years) & 0 \\
$<50$ & +7 \\
$50-60$ & +10 \\
$\geq 60$ & \\
HBV DNA & 0 \\
$\leq 2,000$ & +2 \\
$>2,000$ & \\
FIB-4 & 0 \\
$\leq 3.25$ & +7 \\
$>3.25$ & 0 to 22 \\
\hline Total & \\
\hline
\end{tabular}

HBV, hepatitis B virus; FIB-4, fibrosis-4.
5 years' follow-up duration. The cumulative incidence of HCC was significantly different between low- and high-risk patients by Kaplan-Meier analysis (log-rank test, $P<0.001)$. The annual HCC incidences of low-risk group were less than $0.2 \%$, which is the threshold of offering cost-effective HCC surveillance (Fig. 2A).

We compared the performance of Liang score in patients never receive antiviral treatment and patients received antiviral treatment and censored at the start date of the treatment (Supplementary Table 4). The sensitivity ( $81.82 \%$ vs. $84.38 \%)$ and NPV (99.91\% vs. $99.61 \%)$ were similar between these two groups.

\section{External validation of HCC prediction score}

In the validation cohort, there were 2,686 patients $(62.8 \%)$ classified as low-risk groups, among which three patients (0.11\%) developed HCC. The corresponding sensitivity and NPV of cutoff of 9 were $90.0 \%(95 \% \mathrm{Cl}, 72.3-97.4 \%)$ and $99.9 \%(95 \% \mathrm{Cl}$, 99.6-99.97\%), respectively (Table 5). The cumulative incidence of HCC in low-risk patients was significantly lower than that in highrisk patients (log-rank test, $P<0.001)$, and was less than $0.2 \%$ per year (Fig. 2B). Liang score performed well in patients with or without cirrhosis and subgroups of patients with different AFP levels (Supplementary Tables 5, 6).

\section{Comparison with existing HCC prediction scores}

We compared the performance of Liang score with other HCC prediction scores which were developed in treatment-naïve $\mathrm{CHB}$ patients: CU-HCC score, GAG-HCC score and REACH-B score in the validation cohort. We selected conventionally used cutoff values of 5, 100 and 8 for CU-HCC score, GAG-HCC score and REACH-B

Table 4. Diagnostic accuracy of Liang score (follow-up duration: 5 years)

\begin{tabular}{lccccccc}
\hline & \multicolumn{7}{c}{ Cut off value } \\
\cline { 2 - 8 } & $\mathbf{2}$ & $\mathbf{3}$ & $\mathbf{5}$ & $\mathbf{7}$ & $\mathbf{9}$ & $\mathbf{1 0}$ & $\mathbf{1 2}$ \\
\hline Sensitivity & $97.7(91.1-99.6)$ & $95.3(87.9-98.5)$ & $90.7(82.0-95.6)$ & $89.5(80.6-94.8)$ & $83.7(73.9-90.5)$ & $74.4(63.7-82.9)$ & $55.8(44.7-66.4)$ \\
\hline $\begin{array}{l}\text { Specificity } \\
\text { PPV }\end{array}$ & $19.5(18.8-20.2)$ & $28.8(28.0-29.6)$ & $42.0(41.2-42.9)$ & $48.2(47.3-49.0)$ & $55.4(54.6-56.3)$ & $66.2(65.4-67.0)$ & $80.2(79.5-80.9)$ \\
\hline NPV & $0.8(0.6-1.0)$ & $0.9(0.7-1.1)$ & $1.0(0.8-1.3)$ & $1.1(0.9-1.4)$ & $1.2(1.0-1.6)$ & $1.4(1.1-1.8)$ & $1.8(1.4-2.5)$ \\
$\begin{array}{l}\text { No. of HCC } \\
\text { predicted }\end{array}$ & $99.9(99.7-99.98)$ & $99.9(99.7-99.97)$ & $99.9(99.7-99.9)$ & $99.9(99.7-99.9)$ & $99.8(99.7-99.9)$ & $99.7(99.6-99.8)$ & $99.6(99.5-99.7)$ \\
\hline $\begin{array}{c}\text { No. of low- } \\
\text { risk patients }\end{array}$ & $2,522 / 13,032$ & $3,730 / 13,032$ & $5,451 / 13,032$ & $6,245 / 13,032$ & $7,191 / 13,032$ & $8,592 / 13,032$ & $10,422 / 13,032$ \\
\hline
\end{tabular}

Values are presented as percentage ( $95 \%$ confidence interval) or number.

PPV, positive predictive value; NPV, negative predictive value; HCC, hepatocellular carcinoma. 

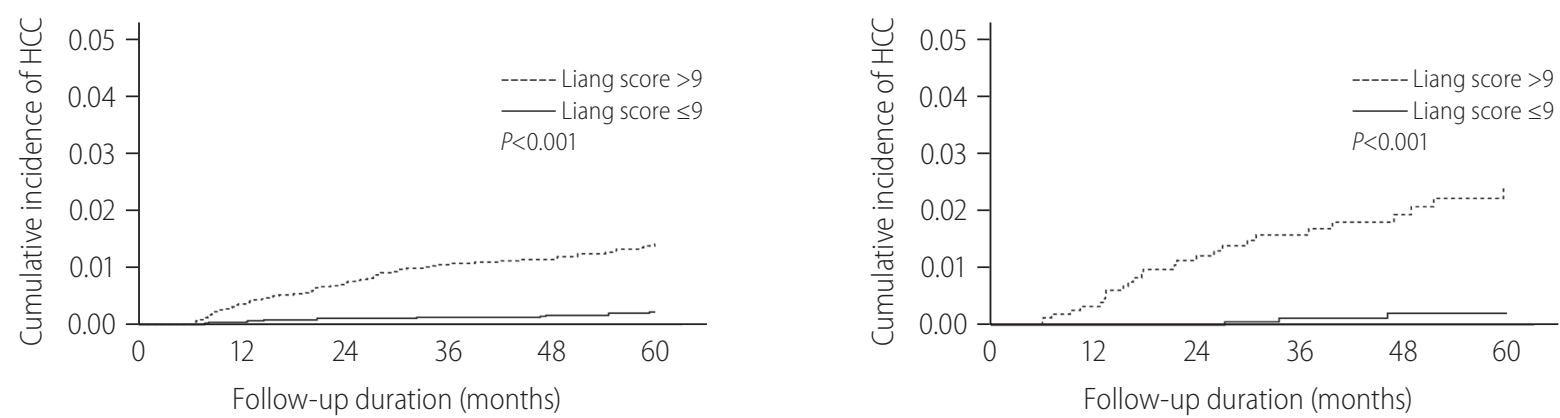

$\begin{array}{lcccccc}\text { No. at risk } & & & & & & \\ \text { Liang score }>9 & 5,841 & 5,738 & 5,394 & 4,652 & 4,025 & 3,406 \\ \text { Liang score } \leq 9 & 7,191 & 7,180 & 6,978 & 6,112 & 5,562 & 5,055 \\ \text { HCC incidence } & - & 0.04 & 0.07 & 0.01 & 0.03 & 0.05\end{array}$

$\begin{array}{lcccccc}\text { No. at risk } & & & & & & \\ \text { Liang score }>9 & 1,594 & 1,458 & 1,208 & 930 & 728 & 547 \\ \text { Liang score } \leq 9 & 2,686 & 2,371 & 1,936 & 1,465 & 1,137 & 883 \\ \text { HCC incidence } & - & 0 & 0 & 0.10 & 0.07 & 0 \\ \text { (\% peryear) } & & & & & & \end{array}$
(\% per year)

B

Figure 2. Cumulative incidence of HCC in the low- and high-risk of patients defined by Liang score in the (A) training cohort and (B) validation cohort with the annual HCC incidence of low-risk patients. HCC, hepatocellular carcinoma.

Table 5. Diagnostic accuracy of Liang score and other HCC prediction scores in the validation cohort (follow-up duration: 5 years)

\begin{tabular}{lcccc}
\hline & Liang score cutoff: $\mathbf{9}$ & CU-HCC score cutoff: $\mathbf{5}$ & GAG-HCC score cutoff: 100 & REACH-B score cutoff: 8 \\
\hline Sensitivity & $90.0(72.3-97.4)$ & $83.3(64.5-93.7)$ & $70.0(50.4-84.6)$ & $65.2(42.8-82.8)$ \\
\hline Specificity & $63.1(61.7-64.6)$ & $57.2(55.7-58.7)$ & $85.3(84.2-86.3)$ & $57.0(55.3-58.6)$ \\
PPV & $1.7(1.1-2.5)$ & $1.4(0.9-2.1)$ & $3.2(2.1-5.0)$ & $1.0(0.6-1.7)$ \\
NPV & $99.9(99.6-99.97)$ & $99.8(99.5-99.9)$ & $99.8(99.5-99.9)$ & $99.6(99.2-99.8)$ \\
No. of HCC predicted & $27 / 30$ & $25 / 30$ & $21 / 30$ & $15 / 23$ \\
No. of patients below cutoff & $2,686 / 4,280$ & $2,403 / 4,219$ & $3,639 / 4,286$ & $1,933 / 3,402$ \\
\hline
\end{tabular}

Values are presented as percentage (95\% confidence interval) or number.

CU-HCC, the Chinese University of Hong Kong-HCC; GAG-HCC, the guide with age, gender, HBV DNA, core promoter mutations and cirrhosis-HCC; REACH-B, the risk estimation for HCC in CHB; PPV, positive predictive value; NPV, negative predictive value; HCC, hepatocellular carcinoma.

score, respectively. ${ }^{11,30,31}$ The sensitivity and NPV results were $83.3 \%$ and $99.8 \%$ for CU-HCC score; $70.0 \%$ and $99.8 \%$ for GAG-HCC score and $65.2 \%$ and $99.6 \%$ for REACH-B score, which were all lower than those of Liang score $(90.0 \%$ and $99.9 \%)$. The number of low-risk patients defined by CU-HCC score, GAG-HCC score and REACH-B score were 2,403, 3,639, and 1,933 with HCC incidence of $0.21 \%, 0.25 \%$, and $0.41 \%$, respectively (Table 5 ). Time-dependent AUROC was used to compare the predictive ability of Liang score with other HCC prediction scores. The time-dependent AUROC of Liang score was $0.79(95 \% \mathrm{Cl}, 0.70-0.89)$ which was not significantly different to those of CU-HCC score (0.82; $95 \% \mathrm{Cl}, 0.75-0.89 ; P=0.434)$ and GAG-HCC score $(0.83$; $95 \% \mathrm{Cl}, 0.74-0.91 ; P=0.342)$. The time-dependent AUROC of Liang score was significantly higher than that of REACH-B score (0.61; $95 \% \mathrm{Cl}, 0.53-0.70 ; P<0.001$; Supplementary Fig. 1).

\section{Patients who may be exempted from HCC surveillance}

Patients with Liang score of 9 or lower may be exempted from HCC surveillance which include the following groups: female patients younger than 50; female patients age between 50 and 60 with low FIB-4 score or male patients younger than 50 with low FIB-4 score (Table 3). The corresponding annual HCC incidences of these groups were all less than $0.2 \%$ in the training cohort.

\section{DISCUSSION}

We aimed to derive and validate a HCC risk score based on serum fibrosis scores to identify a group of patients with very low risk of HCC in treatment-naïve CHB patients. The newly developed 
Liang score was composed of gender, age, HBV DNA and FIB-4 index, which was accurate and reproducible to exclude HCC. By using the cutoff value of 9 , around $50 \%$ of patients were classified as very low risk of HCC (annual incidence $<0.2 \%$ ), so that HCC surveillance would be exempted. As Liang score was composed of common clinical and laboratory parameters, it would be applicable in most clinic settings. This score would contribute to the WHO's hepatitis elimination advocacy by better resource allocation.

The current international guidelines recommend that adult CHB patients with cirrhosis should undergo regular $\mathrm{HCC}$ surveillance. ${ }^{32}$ However, the recommendations in non-cirrhotic CHB patients are not as clear. For example, the American guidelines recommend screening in male CHB patients above 40 years old, which refers to majority of the male patients as adults of early 30 s or younger are mostly vaccinated in our region. With this novel Liang score, the following types of CHB patients would be classified as highrisk with a cutoff value of 9: 1) female patients older than 60 years; 2) female patients with age between 50 and 60 years and FIB-4 $>3.25$; 3) male patients older than 50 years; and 4) male patients younger than 50 years with FIB- $4>3.25$. The corresponding cumulative HCC incidence of these groups at 5 years' follow-up were $1.5 \%, 1.8 \%, 1.7 \%$, and $2.2 \%$ in the training cohort. The annual HCC incidences were all higher than $0.2 \%$ that indicates the possible need of HCC surveillance. These few groups of patients account for $45 \%$ in the training cohort and $37 \%$ in the validation cohort.

A few existing HCC prediction scores such as CU-HCC score and GAG-HCC score include clinical cirrhosis as a key component with strong weight; yet cirrhosis may not be diagnosed accurately by ultrasonography. ${ }^{33}$ We substituted clinical cirrhosis with FIB-4 score, a more objective serum fibrosis score, and then built the Liang score, which is highly applicable and reproducible. By using the cutoff value of 9, Liang score had the highest sensitivity (90.0\%) and NPV (99.9\%) compared with CU-HCC score, GAGHCC score and REACH-B score which were developed in treatment-naive patients. Therefore, Liang score was accurate to rule out HCC in low-risk patients and it worked excellently among current HCC prediction scores in treatment-naïve CHB patients without the necessity of accurate diagnosis of liver cirrhosis.

Our study had some strengths. First, Liang score is composed of common clinical and laboratory parameters such that it is applicable in most clinic settings. Aiming a highly sensitive cutoff achieving very high NPV can identify very-low-risk patients who would not need HCC surveillance in the near future. Second, Liang score is accuracy to exclude low HCC risk patients without the diagnosis of cirrhosis and not affected by operators compared with ultrasound, which is relatively inaccurate to diagnose cirrhosis for HCC surveillance. Third, Liang score was derived in the cohort of Hong Kong patients and externally validated in the Korean cohort. Both of the cohorts had respectable sample size and the results supported that Liang score is accurate and generally applicable in different ethnic groups in Asia.

There were also few limitations of our study. First, Liang score was developed in Hong Kong cohort and validated in Korean cohort, such that its accuracy in Caucasians is uncertain. Further studies based on patients of other ethnic groups are needed. Second, our study was based on treatment-naïve CHB patients. Until further data are available, the findings of our study cannot be extrapolated to patients on antiviral treatment. Third, we did not explore the role of the HBV genotypes as it is not a routine test in Hong Kong and we cannot show the size and stage of HCC. The common HBV genotypes in Hong Kong include genotype $B$ and $C$; whereas it is exclusively genotype $\mathrm{C}$ in Korea. ${ }^{34}$ Genotype C HBV is associated with an increased risk of HCC. ${ }^{34,35}$ Fourth, the HCC incidence of our cohort was only $1.2 \%$ in more than 5 years, as it was a treatment-naïve cohort. Such low incidence further support the urgent need of a highly sensitive score like Liang score which saves the resources of HCC surveillance in around $50 \%$ of such patients. Fifth, there were many missing HBV DNA results in our cohort as the test was available widely in Hong Kong from the year of 2012. Many old patients may did not receive the test. Sixth, we did not collect the presence of non-alcoholic fatty liver disease and other metabolic factors like insulin resistance and obesity which may affect the risk of HCC at long term. Finally, the diagnosis of cirrhosis in our study most based on the ultrasonography, which may be not accurate and influence the reliability of CU-HCC score and GAG-HCC score.

In conclusion, Liang score, composed of common parameters including gender, age, HBV DNA and FIB-4 index, is accurate to exclude HCC in treatment-naïve CHB patients. Liang score identifies patients at very low risk of HCC so that HCC surveillance can be exempted. Further studies are needed to validate the Liang score in prospective cohorts.

\section{Authors' contribution}

All authors were responsible for the study concept and design. Lilian Liang, Yee-Kit Tse, Terry Yip and Grace Wong were responsible for the acquisition and analysis of data, had full access to all of the data in the study and take responsibility for the integrity of 
the data and the accuracy of the data analysis. All authors were responsible for the interpretation of data, the drafting, and critical revision of the manuscript for important intellectual content.

\section{Acknowledgements}

This work was supported by the Health and Medical Research Fund (HMRF) of the Food and Health Bureau (Reference no: 15160551).

\section{Conflicts of Interest}

Vincent Wong has served as an advisory committee member for AbbVie, Allergan, Gilead Sciences, Janssen, Perspectum Diagnostics and Pfizer; and a speaker for Bristol-Myers Squibb, Echosens, Gilead Sciences and Merck.

Terry Yip has served as an advisory committee member and speaker for Gilead Sciences.

Henry Chan is an advisor for AbbVie, Aptorum, Altimmune, Arbutus, Intellia, Janssen, Gilead, GRAIL, Medimmune, Roche, Vir Biotechnology; and a speaker for AbbVie, Gilead and Roche.

Grace Wong has served as an advisory committee member for Gilead Sciences and Janssen, as a speaker for Abbott, Abbvie, Ascletis, Bristol-Myers Squibb, Echosens, Furui, Gilead Sciences, Janssen and Roche, and received research grant from Gilead Sciences.

The other authors have no conflicts to disclose.

\section{SUPPLEMENTARY MATERIAL}

Supplementary material is available at Clinical and Molecular Hepatology website (http://www.e-cmh.org).

\section{REFERENCES}

1. Polaris Observatory Collaborators. Global prevalence, treatment, and prevention of hepatitis B virus infection in 2016: a modelling study. Lancet Gastroenterol Hepatol 2018;3:383-403.

2. Singal $A G$, Lampertico $P$, Nahon P. Epidemiology and surveillance for hepatocellular carcinoma: new trends. J Hepatol 2020;72:250-261.

3. Wong GL, Wong VW, Tan GM, Ip KI, Lai WK, Li YW, et al. Surveillance programme for hepatocellular carcinoma improves the survival of patients with chronic viral hepatitis. Liver Int 2008;28:79-87.

4. Terrault NA, Lok ASF, McMahon BJ, Chang KM, Hwang JP, Jonas $M M$, et al. Update on prevention, diagnosis, and treatment of chronic hepatitis B: AASLD 2018 hepatitis B guidance. Hepatology 2018:67:1560-1599.

5. European Association for the Study of the Liver. EASL 2017 clinical practice guidelines on the management of hepatitis B virus infection. J Hepatol 2017;67:370-398.

6. Sarin SK, Kumar M, Lau GK, Abbas Z, Chan HL, Chen CJ, et al. Asian-Pacific clinical practice guidelines on the management of hepatitis B: a 2015 update. Hepatol Int 2016;10:1-98.

7. Wong GL, Chan HL, Mak CW, Lee SK, Ip ZM, Lam AT, et al. Entecavir treatment reduces hepatic events and deaths in chronic hepatitis B patients with liver cirrhosis. Hepatology 2013;58:1537-1547.

8. Yip TC, Wong VW, Chan HL, Tse YK, Lui GC, Wong GL. Tenofovir is associated with lower risk of hepatocellular carcinoma than entecavir in patients with chronic HBV infection in China. Gastroenterology 2020;158:215-225.e6.

9. Bonacci M, Lens S, Mariño Z, Londoño MC, Rodríguez-Tajes S, Mas A, et al. Anti-viral therapy can be delayed or avoided in a significant proportion of HBeAg-negative Caucasian patients in the grey zone. Aliment Pharmacol Ther 2018;47:1397-1408.

10. Sinn DH, Lee J, Goo J, Kim K, Gwak GY, Paik YH, et al. Hepatocellular carcinoma risk in chronic hepatitis $B$ virus-infected compensated cirrhosis patients with low viral load. Hepatology 2015;62:694-701.

11. Wong VW, Chan SL, Mo F, Chan TC, Loong HH, Wong GL, et al. Clinical scoring system to predict hepatocellular carcinoma in chronic hepatitis B carriers. J Clin Oncol 2010;28:1660-1665.

12. Wong GL, Chan HL, Wong CK, Leung C, Chan CY, Ho PP, et al. Liver stiffness-based optimization of hepatocellular carcinoma risk score in patients with chronic hepatitis B. J Hepatol 2014;60:339-345.

13. Wong VW, Wong GL, Chim AM, Choi PC, Chan AW, Tsang SW, et al. Surrogate end points and long-term outcome in patients with chronic hepatitis B. Clin Gastroenterol Hepatol 2009;7:1113-1120.

14. Liang LY, Wong VW, Tse YK, Yip TC, Lui GC, Chan HL, et al. Improvement in enhanced liver fibrosis score and liver stiffness measurement reflects lower risk of hepatocellular carcinoma. Aliment Pharmacol Ther 2019;49:1509-1517.

15. Cheng JYK, Wong GLH. Advances in the diagnosis and treatment of liver fibrosis. Hepatoma Res 2017;3:156-169.

16. Wong GL, Wong VW, Choi PC, Chan AW, Chan HL. Development of a non-invasive algorithm with transient elastography (Fibroscan) and serum test formula for advanced liver fibrosis in chronic hepatitis B. Aliment Pharmacol Ther 2010;31:1095-1103.

17. Brouwer WP, van der Meer AJP, Boonstra A, Plompen EPC, Pas SD, de Knegt RJ, et al. Prediction of long-term clinical outcome in a diverse chronic hepatitis B population: role of the PAGE-B score. J Viral Hepat 2017;24:1023-1031.

18. World Health Organization (WHO). Combating hepatitis B and C to reach elimination by 2030: advocacy brief. WHO web site, <https:// apps.who.int/iris/handle/10665/206453>. Accessed 17 Jun 2021. 
19. Cheng M, Tong Y, Kwok T, Chenga I, Chunga A, Leunga J, et al. Development journey of clinical data analysis and reporting system (CDARS) in hospital authority of Hong Kong. MEDINFO 2010;160:1468.

20. Yip TC, Wong GL, Wong VW, Tse YK, Lui GC, Lam KL, et al. Durability of hepatitis $B$ surface antigen seroclearance in untreated and nucleos(t)ide analogue-treated patients. J Hepatol 2018;68:63-72.

21. Wong GL, Chan HL, Tse YK, Yip TC, Lam KL, Lui GC, et al. Chronic kidney disease progression in patients with chronic hepatitis $B$ on tenofovir, entecavir, or no treatment. Aliment Pharmacol Ther 2018:48:984-992.

22. Yip TC, Chan HL, Tse YK, Lam KL, Lui GC, Wong VW, et al. On-treatment improvement of MELD score reduces death and hepatic events in patients with hepatitis B-related cirrhosis. Am J Gastroenterol 2018;113:1629-1638.

23. Lai JC, Wong GL, Yip TC, Tse YK, Lam KL, Lui GC, et al. Chronic hepatitis $B$ increases liver-related mortality of patients with acute hepatitis E: a territorywide cohort study from 2000 to 2016. Clin Infect Dis 2018;67:1278-1284.

24. Wong JC, Chan HL, Tse YK, Yip TC, Wong VW, Wong GL. Statins reduce the risk of liver decompensation and death in chronic viral hepatitis: a propensity score weighted landmark analysis. Aliment Pharmacol Ther 2017;46:1001-1010.

25. Vallet-Pichard A, Mallet V, Nalpas B, Verkarre V, Nalpas A, DhalluinVenier $\mathrm{V}$, et al. FIB-4: an inexpensive and accurate marker of fibrosis in HCV infection. Comparison with liver biopsy and fibrotest. Hepatology 2007;46:32-36.

26. Castéra L, Vergniol J, Foucher J, Le Bail B, Chanteloup E, Haaser M, et al. Prospective comparison of transient elastography, Fibrotest, APRI, and liver biopsy for the assessment of fibrosis in chronic hepatitis C. Gastroenterology 2005;128:343-350.
27. Heagerty PJ, Lumley T, Pepe MS. Time-dependent ROC curves for censored survival data and a diagnostic marker. Biometrics 2000;56:337-344.

28. Wong GL, Wong VW, Yuen BW, Tse YK, Luk HW, Yip TC, et al. An aging population of chronic hepatitis $B$ with increasing comorbidities: a territory-wide study from 2000 to 2017. Hepatology 2020;71:444-455.

29. Yotsuyanagi H, Takano T, Tanaka M, Amano K, Imamura M, Ogawa $\mathrm{K}$, et al. Hepatitis $B$ virus-related hepatocellular carcinoma in young adults: efficacy of nationwide selective vaccination. Hepatol Res 2020;50:182-189.

30. Yuen MF, Tanaka Y, Fong DY, Fung J, Wong DK, Yuen JC, et al. Independent risk factors and predictive score for the development of hepatocellular carcinoma in chronic hepatitis B. J Hepatol 2009;50:8088.

31. Wong GL, Wong VW. Risk prediction of hepatitis B virus-related hepatocellular carcinoma in the era of antiviral therapy. World J Gastroenterol 2013;19:6515-6522.

32. Marrero JA, Kulik LM, Sirlin CB, Zhu AX, Finn RS, Abecassis MM, et al. Diagnosis, staging, and management of hepatocellular carcinoma: 2018 practice guidance by the American Association for the Study of Liver Diseases. Hepatology 2018;68:723-750.

33. Ong TZ, Tan HJ. Ultrasonography is not reliable in diagnosing liver cirrhosis in clinical practice. Singapore Med J 2003;44:293-295.

34. Wong GL, Chan HL, Yiu KK, Lai JW, Chan VK, Cheung KK, et al. Meta-analysis: the association of hepatitis $B$ virus genotypes and hepatocellular carcinoma. Aliment Pharmacol Ther 2013;37:517526.

35. Chan HL, Hui AY, Wong ML, Tse AM, Hung LC, Wong VW, et al. Genotype $C$ hepatitis $B$ virus infection is associated with an increased risk of hepatocellular carcinoma. Gut 2004;53:1494-1498. 